\title{
Living with Merkel Cell Carcinoma (MCC): Development of a Conceptual Model of MCC Based on Patient Experiences
}

\author{
Howard L. Kaufman ${ }^{1,5}$ - Carla Dias Barbosa ${ }^{2}$ Isabelle Guillemin ${ }^{2}$. \\ Jérémy Lambert $^{2} \cdot$ Lisa Mahnke $^{3} \cdot$ Murtuza Bharmal $^{4}$
}

Published online: 6 March 2018

(C) The Author(s) 2018. This article is an open access publication

\begin{abstract}
Background Merkel cell carcinoma (MCC) is a rare and aggressive skin cancer with limited treatment options at advanced stages. There is a paucity of data available regarding the impact of MCC and its management on patients' lives. This study aimed to address this gap by interviewing patients with metastatic MCC entering a trial of an immunotherapy (avelumab).

Methods In a single-arm, open-label, international, phase 2 trial in patients with stage IV, chemotherapy-refractory, histologically confirmed MCC, patients were invited to participate in semi-structured phone interviews. These were conducted before avelumab administration. Interview transcripts were analysed qualitatively to identify concepts important to patients relating to their experience of metastatic MCC and its management.

Results Nineteen patients were interviewed. Most reported MCC to be painless and asymptomatic. They reported being often misdiagnosed and described a long process before receiving the correct diagnosis. They also reported a feeling of "shock" after being informed of the severity and seriousness of their cancer. Overall, patients did not report impaired physical and cognitive capacities or impact on daily lives, either before or after diagnosis. However,
\end{abstract}

Howard L. Kaufman

hk553@cinj.rutgers.edu

1 Rutgers Cancer Institute of New Jersey, New Brunswick, NJ, USA

2 Patient-Centered Outcomes, Mapi, Lyon, France

3 EMD Serono, Boston, USA

4 Merck KGaA, Darmstadt, Germany

5 Present Address: Replimune Inc, Woburn, MA, USA patients and their relatives reported feelings of "worry" and "fear" about the unknown outcome of the disease. Chemotherapy and radiotherapy negatively affected patients physically and psychologically in their everyday lives.

Conclusions MCC disease was not perceived by the interviewed patients to result in severe functional limitations or to severely impact everyday activities, but was associated with substantial negative psychological impact. In contrast, chemotherapy and radiotherapy for MCC are highly debilitating and disrupt patients' lives. ClinicalTrials.gov identifier NCT02155647.

\section{Key Points for Decision Makers}

MCC is painless and symptomless for most patients, both prior to diagnosis and during the journey.

Overall, living with MCC is close to normal in terms of daily functioning; patients are still able to perform their activities of daily living and still have all their physical capabilities. In contrast, MCC has a substantial psychological impact on the patients and their relatives and friends; in most cases, patients are scared of the unknown and the future.

Patients described chemotherapy and radiotherapy as highly debilitating, both physically and mentally. 


\section{Introduction}

Merkel cell carcinoma (MCC) is a rare and aggressive skin cancer that is challenging to treat in metastatic stages. The disease usually appears as a single painless papule on sunexposed skin, often with benign appearance, leading to delays in biopsies. Factors strongly associated with the development of MCC include older age (more than 65 years), skin phenotype, immune suppression, and history of extensive sun exposure $[1,2]$. The tumour is often associated with DNA integration of the unique Merkel cell virus, a polyomavirus, or the presence of a high rate of UVassociated DNA mutations [3, 4], both of which have been associated with $\mathrm{T}$-cell responses.

Although few epidemiological data have been published on this rare carcinoma, recent publications have shown that the incidence of MCC is increasing rapidly in the USA and Europe [5-7]. Surgery is the standard treatment for early stage MCC. Historically, there have been no approved treatments for recurrent, late-stage (advanced or metastatic), non-resectable MCC. However, in 2017, avelumab was approved both in the USA and in Europe for the treatment of patients 12 years and older with metastatic MCC. It is thus the first available treatment option in this indication. For recurrent, late-stage patients, the National Comprehensive Cancer Network guidelines recommend a multidisciplinary clinical team to consider the following treatment options: radiotherapy, surgery, systemic therapies or chemotherapy, or a combination of some or all of these. They further promote participation in a clinical trial as an option for such patients to receive innovative treatments [8]. Although MCC is considered chemosensitive, it is associated with a short duration of response with a high recurrence rate, including in early disease stages [9]. The recent European guidelines describe chemotherapy responses as being "of short duration with a median overall survival rate of 9 months and high toxicity in elderly patients" [10].

Avelumab is a human monoclonal antibody that binds and inhibits programmed death ligand 1. The use of immunotherapy in MCC is new and provides a distinct mechanism of action and adverse event profile compared with cytotoxic chemotherapy, which has been the standard treatment in these patients. The safety and efficacy of this immunotherapy was assessed in a phase 2 trial (JAVELIN Merkel 200; NCT02155647) in 88 patients with stage IV chemotherapy-refractory MCC and was associated with durable responses in $32 \%$ of patients [11]. In addition, pembrolizumab, a programmed cell death receptor 1 (PD1)-targeted monoclonal antibody, has also shown promise in patients with metastatic MCC treated in the first-line setting [12]. These developments have supported immunotherapy as a new standard of care for patients with advanced and metastatic MCC.

While the availability of new therapeutic options is welcome, there is a paucity of data available regarding the impact of MCC disease and its management on patients' health-related quality of life. Therefore, qualitative research was undertaken to provide a comprehensive overview of the impact of MCC and its management on patients' lives prior to diagnosis, at diagnosis, and after diagnosis, and to develop a disease model of adults with metastatic MCC. Qualitative exploratory interviews were conducted with the patients enrolled in the JAVELIN Merkel 200 clinical trial, at baseline before the start of the study treatment. In this manuscript, we report the methodology and findings of this qualitative research.

\section{Materials and Methods}

\subsection{Study Design}

The single-arm, open-label, multicentre, international, phase 2 JAVELIN Merkel 200 trial was conducted to evaluate the efficacy and safety of avelumab in patients with stage IV, chemotherapy-refractory, histologically confirmed MCC (aged $\geq 18$ years), i.e. metastatic patients who had failed at least one line of chemotherapy (NCT02155647). Details of the design of the trial, including efficacy and safety endpoints, have been reported elsewhere [11]. Upon recruitment, patients were invited to participate in optional qualitative interviews in all countries (Australia, Austria, Canada, France, Germany, Italy, Spain, Switzerland, and the USA), except Japan where interviews were viewed as culturally insensitive. Patients agreeing to participate were interviewed during the screening period, before first administration of the study treatment (baseline patient interviews).

The clinical trial protocol, including description of the qualitative interviews, was approved by all relevant independent ethics committees and institutional review boards and was conducted in accordance with the Declaration of Helsinki and Good Clinical Practice. Patients provided written informed consent before any trial-related activity. Patients who agreed to be interviewed indicated their willingness to participate within the informed consent form.

\subsection{Baseline Patient Interviews}

Thirty-minute exploratory phone interviews were conducted by experienced psychologists/researchers external to the clinical teams and specifically trained on this project. Telephone interviews were used to allow patients across a 
wide geographical scale to be interviewed and to allow for easy and flexible scheduling and rescheduling of interviews according to patients' status. Comparative studies have suggested that the content and the quality of phone interviews could be comparable to those of face-to-face interviews [13, 14]. The interviewers used open-ended questions to elicit spontaneous concepts relevant to the patients, including living with MCC, their experience of the disease and its management, and its impact on their everyday lives prior to diagnosis (e.g. What were the symptoms at first? What was your reaction when you were diagnosed?), at and after diagnosis (e.g. Did the cancer change your everyday life? What kind of daily activities were affected? How? How would you describe your emotional state, your mood at this time?), and during treatment with chemotherapy and/or radiotherapy [e.g. Do you know what treatment for your cancer you received prior to entering in the study, if any (e.g. chemotherapy, radiation, surgery)? Did you perceive or feel any benefits from this treatment?]. To help the interviewer conduct the interview, a semi-structured, study-specific guide was developed that contained the themes to be addressed: (1) patients' experience from the onset of the disease to the time of diagnosis, including initial symptoms and the patients' journey up to diagnosis; (2) patients' experience of the disease over the time from diagnosis to the time of the interview, including the impact on patients' psychological, physical, and social well-being; and (3) patients' experience of previously received chemotherapy and/or radiotherapy and the impact on patients' daily routines. Non-directive interview techniques were used to allow interviewees to answer spontaneously, and probes were used only to collect additional details when applicable and informative. Interviews were audio-recorded and transcribed verbatim. To ensure patient confidentiality, any information that could identify a patient was removed from the transcripts prior to the qualitative analysis.

\subsection{Qualitative Analysis of Patient Interviews}

Interview transcripts were analysed by trained and experienced qualitative researchers (JL, IG), using the ATLAS.ti qualitative software package [15, 16] and a thematic analysis [17]. JL, who is a male researcher holding a PhD in oncology, analysed all interviews; IG, a female researcher with many years of experience in qualitative research, analysed separately about two thirds of the transcripts $(n=11)$. Both researchers discussed their analyses and solved any conflicting points, before aggregating the results and drawing conclusions. The analysis consisted of the attribution of tags (a code made of one to two words) summarising the idea being conveyed to a word, a sentence, or a paragraph of each of the transcripts.
A first specific tag was applied related to the time of reference (e.g. prior to diagnosis, at diagnosis). Second-level tags corresponded to the general concept reported by the patients, e.g. symptoms and impacts (physical, emotional, cognitive, etc.). "Descriptors" constituted the third level and potentially fourth level of tags and were intended to help the researcher to specifically define each symptom or impact experienced by a patient with MCC. The coding process was iterative and interpretive, with constant comparison and interaction between codes and transcripts, thus allowing the codes to be renamed and enriched with descriptors to bring more precision. Patient quotations were compared and contrasted iteratively by comparing earlier and later interviews and by concepts, e.g. are "cramping" and "abdominal pain" the same concept? A disease conceptual model of MCC was then developed from the concepts and sub-concepts elicited, allowing a representation of the journey and everyday life experience of a patient with MCC, from prior to diagnosis to after being diagnosed, up to the time of the interview [18].

\section{Results}

\subsection{Patient Characteristics}

Between 25 July 2014 and 3 September 2015, 19 patients (mean age 72 years, $79 \%$ male) out of 88 patients enrolled in the trial were interviewed before they received the first dose of avelumab and were included in the analysis. All interviewed patients consented to participation in the study. Seventeen patients were from the USA, and two were from Germany. Most patients were retired (84\%) and were living with a spouse or partner $(89 \%)$ (Table 1$)$.

\subsection{Qualitative Analysis of Patient Interviews}

The qualitative analysis of interviews allowed the patients' experience and perceptions of their disease and its management to be assessed. Findings from this analysis are detailed below.

\subsubsection{Diagnostic Process and Length of Time to Diagnosis}

When describing their journey from initial presentation to being diagnosed with MCC, patients said that their physicians, general practitioners, and dermatologists, and occasionally their oncologists, had a lack of knowledge about MCC. Consequently, most patients $(n=10)$ reported they were referred many times to different doctors and underwent many tests prior to the diagnosis. In parallel, several patients $(n=7)$ reported that they had initially been misdiagnosed. Similarly, some patients $(n=2)$ believed they 
Table 1 Baseline demographic and clinical characteristics of interviewed patients

\begin{tabular}{ll}
\hline Characteristics & Value $(n=19)$ \\
\hline Age, years & \\
Mean (SD) & $72.2(8.2)$ \\
Median (min-max) & $72.0(55.0-85.0)$ \\
Gender, $n(\%)$ & $15(78.9 \%) / 4(21.1 \%)$ \\
Male/female & \\
Country, $n(\%)$ & $2(10.5 \%)$ \\
Germany & $17(89.5 \%)$ \\
USA & \\
Living status, $n(\%)$ & $17(89.5 \%)$ \\
Living with spouse/partner & $2(10.5 \%)$ \\
Widow & $1(5.3 \%)$ \\
Occupation, $n(\%)$ & $2(10.5 \%)$ \\
Full-time working & $16(84.2 \%)$ \\
Not working/on leave & \\
Retired & $10(52.6 \%)$ \\
ECOG performance status score & $9(47.4 \%)$ \\
0 & $83.3(49.9)$ \\
1 & $78.5(16.0-182.0)$ \\
Tumour size at baseline, mm & 3 \\
Mean (SD) & $2.3(0.8)$ \\
Median (min-max) & $2.0(1.0-3.0)$ \\
Missing data & $S D$ \\
Time since initial diagnosis, years & \\
Mean (SD) & \\
Median (min-max) & \\
\hline
\end{tabular}

ECOG Eastern Cooperative Oncology Group, SD standard deviation

had a "simple infection" or "a spot" and not a cancer before being diagnosed with MCC.

According to patients, the diagnosis of MCC was very often made on the basis of the biopsy results.

Overall, patients highlighted that they could not obtain much information about MCC from the internet and healthcare professionals $(n=3)$ or that information available on the internet was scary $(n=3)$.

Patients' quotes related to the diagnostic process are provided in Table 2.

\subsubsection{Symptoms and Impact of MCC on Patients' Lives}

Patients described MCC as a "very serious", "aggressive", "rare", and/or "scary" disease that they first noticed as a bump growing on a part of their body.

All but one patient used the words "lump" and "bump" to describe the first sign of their cancer; other words that were used included "cyst", "nodules", or "spots". Five patients noted that they were aware of the lump for months or even years before it began to progress rapidly.

Most patients spontaneously reported experiencing very little or no pain $(n=9)$ or symptoms $(n=2)$ at the time of diagnosis or before diagnosis in relation to MCC. Pain was only mentioned spontaneously by some patients in relation to the surgery received to remove the tumour.

The absence of pain and symptoms associated with MCC was also reported when patients were asked to consider the period after diagnosis.

The impact of MCC on patients' lives started at the moment of diagnosis, with an emotional reaction to diagnosis. Most patients $(n=7)$ said they did not know what to expect or the consequences of being diagnosed with MCC; three described their first reaction as mainly confusion and disbelief. They also understood from their doctor that they had to be treated quickly.

Once the patients became aware of the seriousness of the cancer, they then reported feelings of "shock" $(n=8)$, "despair" $(n=3)$, "fear" $(n=2)$, and "worry" $(n=5)$ about the future and the unknown. Two patients took the diagnosis philosophically or spiritually.

Patients $(n=4)$ reported that families and relatives also shared the concerns, worries, and sadness.

Most patients did not have their daily routine (driving, social life, and cognitive abilities) affected by MCC, whether prior to diagnosis, at diagnosis, or after diagnosis. This was reported both spontaneously and after being probed. Patients mentioned that their lives were not disrupted by the disease itself, and they did not have to depend on others. They felt physically "fine" and "in really good shape".

Only two patients mentioned an impact of MCC on their everyday lives, but this was more due to the consequences of surgical resection of the tumour and the associated recovery period, rather than the cancer itself.

While five patients did not report having sleep problems, four mentioned that they had trouble sleeping due to anxiety related to diagnosis, and others did not know the reason for their sleep problems.

Patients often had difficulty in linking pain or other effects to MCC disease, treatment, or both. However, fatigue and lack of energy were systematically linked to treatment, particularly chemotherapy.

In contrast, psychological aspects of patients' lives were substantially affected. Patients $(n=6)$ reported being "worried" and "feeling down" about the unknown future and the disease; several patients said they were "scared". Some patients $(n=3)$ also reported being affected seeing their loved ones feeling worried and sad, as well as thinking about their relatives' future without them. 
Table 2 Selected patient quotes related to diagnostic process and length of time to diagnosis

\begin{tabular}{ll}
\hline Concepts & Illustrative patient quotes \\
\hline $\begin{array}{l}\text { Several tests and } \\
\text { visits }\end{array}$ & "The dermatologist referred me then to an oncologist but the oncologist didn't know anything about it either so he referred \\
me to [XXX] Cancer Center, to a doctor at the [XXX] Cancer Center" (patient 2) \\
Misdiagnosis & "She looked at it and felt it and said 'Oh, it was really pretty small,' she said 'I think it's just some fatty tissue, I don't think \\
& it's anything to worry about" (patient 11) \\
& "I thought it was the beginnings of an infection, you know, I thought I had, infection related, that's why the lymph node \\
& was reacting so I went to the doctor for a consultation on it" (patient 1) \\
"It's like, there isn't a lot of information out there" (patient 2) \\
$\begin{array}{l}\text { Lack of } \\
\text { information }\end{array}$
\end{tabular}

However, one third of patients stated that they were trying to remain in a positive frame of mind to fight the disease.

The situation also affected patients' family lives, mostly psychologically. Patients described their family and relatives as feeling "worried", "upset", "hurt" $(n=9)$, and "not dealing well with it" $(n=2)$, but providing support both morally and practically in terms of daily routines and coordinating and managing the patients' care for most of them $(n=14)$.

Some patients $(n=4)$ also mentioned that the disease had strengthened their relationships with family, relatives, and friends, which help them discuss their feelings more openly.

Patients' quotes related to the symptoms and the impact of MCC are provided in Table 3.

\subsubsection{Patients' Perceptions of Chemotherapy and Radiotherapy and Expectations Toward the New Study Treatment}

All interviewed patients reported having received chemotherapy following surgery, and 14 reported also receiving radiotherapy. In most cases treatment was initiated rapidly after diagnosis. Patients reported varying perceptions about the efficacy of chemotherapy and radiotherapy. The majority of patients $(n=12)$ noted that chemotherapy was successful at first, but did not have a long-lasting effect, resulting in cancer recurrence within weeks or months after stopping treatment.

Seven patients reported an improvement in their tumour of either a shrinkage in lymph nodes or a reduction in the number of tumours.

Four patients did not report any efficacy associated with chemotherapy, and some reported a worsening of MCC symptoms.

Overall, patients described an efficacy pattern with radiotherapy that was similar to chemotherapy, with mixed reports about efficacy in preventing recurrence, or no efficacy.

Similarly, patients had varying experiences in terms of the tolerability of treatments. Most side effects were reported only when the patients were probed (i.e. when answering the question "Did you experience any side effects with this treatment?"). One third of patients spontaneously reported having no or very few side effects. Fatigue, exhaustion, and lack of energy were the most frequently reported side effects among the 12 patients who reported chemotherapy-related side effects:

Nine patients reported "nausea" and/or "vomiting", five reported "appetite problems", and three reported "hair loss", "blood clots", and "numbness in the hands". Other side effects mentioned by one or two patients included "weight loss" or "weight gain", "hearing loss", "decreased blood pressure", and "breast swelling".

Some patients reported side effects of radiotherapy, most frequently burns $(n=4)$; four patients reported no side effects of radiotherapy.

The impact of chemotherapy on patients' lives was substantial. Patients described it as "very disruptive" (patient 3), "debilitating" (patient 9), "devastating" (patient 1), "poison" (patient 5), and "nasty stuff" (patient 11); three patients reported that the treatment had a deleterious impact on their daily activities. The most common impact was due to fatigue and lack of energy resulting from treatment $(n=8)$; patients reported feeling slowed down, having to rest more, or being limited in their capacities.

Three patients reported an adverse impact also on sleep.

Six patients reported no negative effect of treatment or very little change in their quality of life and everyday lives.

Three patients complained about the chemotherapy schedule being time consuming or constraining.

When specifically probed, patients reported little direct impact of radiotherapy on their daily lives; instead, this was linked to the constraints of having to go to the hospital, spending time in hospital, and planning for hospital appointments $(n=5)$. 
Table 3 Selected patient quotes related to symptoms and impact of MCC

\begin{tabular}{|c|c|}
\hline Concepts & Illustrative patient quotes \\
\hline Growing lump & $\begin{array}{l}\text { "It quickly grew, over a month, month and a half, it really just, like a volcano is the way I describe it" } \\
\text { (patient 16) } \\
\text { "I had a bump on my upper chest, I guess right by my neck" (patient 4) } \\
\text { "If you'd like a little background that goes back maybe several years, I had a small growth on my back that } \\
\text { my doctors were watching for several years, and it suddenly, after many years, turned into a growth that } \\
\text { was growing at a very rapid pace" (patient 5) }\end{array}$ \\
\hline Painless & $\begin{array}{l}\text { "It's silent" (patient 8); "The cancer doesn't hurt!" (patient 3) } \\
\text { "I wasn't in any pain or anything, well there was pain associated with where the... actually the pain was } \\
\text { where the graft sites on my legs were, but I think that may have been as a result of my clothes rubbing } \\
\text { against it (patient 10) }\end{array}$ \\
\hline Shocked, scared by the diagnosis & $\begin{array}{l}\text { "I didn't know what to think because I didn't know what it was" (patient 12) } \\
\text { "I was, like anybody would be, I was just like a deer in the headlights, you know, I didn't know, you know, } \\
\text { what's gonna happen here!" (patient 16) }\end{array}$ \\
\hline $\begin{array}{l}\text { Unaffected daily routine and } \\
\text { physical condition }\end{array}$ & $\begin{array}{l}\text { "I don't need any daily help. [...] Because I'm not disabled one bit, ma'am" (patient 8) } \\
\text { "We went on holiday, and during that period I was still riding a bicycle" (patient 18) } \\
\text { "The cancer, then or now, has not affected me at all. You know, if you looked, if you walked up to meet } \\
\text { me you would not know I had cancer. I don't have you know, this is, I hate to say it this way, but you } \\
\text { know, this is the only way to say it, a lot of people with cancer have a look, you know? And they become } \\
\text { frail, weak looking, their face gets something in it. I never got that way. You know, I mean, I never had } \\
\text { that type of look" (patient 3) }\end{array}$ \\
\hline $\begin{array}{l}\text { Anxiety-related sleeping } \\
\text { difficulties }\end{array}$ & $\begin{array}{l}\text { "I'll tell you one thing I'm a very bad sleeper and it got worse. [...] It got worse, only because of my } \\
\text { anxiety" (patient 15) } \\
\text { "I can tell you that before I had cancer, I could sleep very well, to be honest with you, you know, I mean I } \\
\text { could go to bed anywhere between 9:30 and 10:30 at night and I could sleep until 6:30 in the morning, } \\
\text { and never move, and then, I don't know if the cancer had any effect, the treatment had any effect, or } \\
\text { what... I haven't had a full week's sleep since I have been diagnosed with cancer" (patient 3) }\end{array}$ \\
\hline Fighting the disease & $\begin{array}{l}\text { "Most of, the thing is, I think, in this battle with cancer, is to stay moving forward, stay positive and that's } \\
\text { what I've tried to do and that's what I am going to continue to do, to keep my positive outlook that } \\
\text { something's going to work" (patient 1) }\end{array}$ \\
\hline Supportive relatives and friends & $\begin{array}{l}\text { "They've been most supportive, my wife has just been a sweetheart, she's been with me every step, she sat } \\
\text { through every chemo treatment with me, and got me stuff to sip, and crunch on and snack on, and she's } \\
\text { always been there with me, all the doctors' appointments, the oncologist's appointments, she's always sat } \\
\text { in with me" (patient 11) } \\
\text { "It probably made me let them know how much I felt about them more, and made me tell them more often } \\
\text { how I felt about them, than not, it didn't have any adverse effect, it brought us closer together" (patient } \\
\text { 3) } \\
\text { "They would all come over, they live, my children live about two hours away, and they made extra special } \\
\text { efforts to spend more time, and they would come over" (patient 17) }\end{array}$ \\
\hline
\end{tabular}

MCC Merkel cell carcinoma

Four patients reported no impact of radiotherapy on daily activities.

Almost all patients had strong hopes on entering the trial and receiving avelumab, the study treatment. Patients unanimously hoped to extend their life by some months, hoped to be cured, or hoped for any positive results. A couple of patients disclosed that they were excited and happy about starting the trial and receiving avelumab, even though being a little anxious about not knowing the future. According to patients' responses to the question "What do you know about the study treatment?", the large majority had understood that the study drug involved or stimulated their immune system.
Patients' quotes related to their experience of both chemotherapy and radiotherapy for the treatment of MCC, and their expectations towards the study treatment are provided in Table 4.

\subsection{Development of the Conceptual Model of the Patients' Experience with MCC and Its Management}

Based on the findings from the qualitative analysis, the codes were organised into a conceptual model representing the patients' journey with MCC and its management, from before diagnosis to the time of trial enrolment (Fig. 1). 
Table 4 Selected patient quotes related to patients' experience with chemo and radiotherapy and expectations toward the study treatment

Concepts Illustrative patient quotes

Limited perceived efficacy of chemotherapy: success/relapse/failure

Limited perceived efficacy of radiotherapy: success/relapse/failure

Tolerability issues

Disrupted activities

Expectations towards the study treatment
"We were getting some fairly dramatic results in tumor shrinkage and I really, even though it was awful to have to take the medicine, it was kind of bitter sweet, because it was in fact reducing the size of the tumors" (patient 5)

"That was the 2nd time I had chemo, and I had the lumps, or whatever you want to call them, and they all disappeared, they all shrunk, and after that, a month or so later, more popped up. Now. I've got them all over, so, that's how I'm sitting right now" (patient 8)

"They gave me three sessions of chemotherapy and the tumors disappeared, but they only stayed off for about 2 or 3 weeks then the tumors came back. I think I was kind of down in the dumps after that, I thought we had licked it, and I got, you know, I was boosted, my morale was pretty high because the, er, tumors went away, but then I was not so happy that they came back" (patient 10)

"Only the benefit that the tumor shrunk for three months. And then it started to grow again" (patient 15)

“No, not for me it wasn't, it never slowed them down, they just kept coming you know, these new tumors" (patient 11)

"I did feel good about it (radiotherapy) because everything seemed to stabilize and, I was not bothered by this cancer for I guess, well about a year" (patient 10)

"I went to radiology for, I had, I can't remember, about 30 sessions of radiation, in that area. Like I say I was good for a month or so after that, I'm talking in general terms, and then like I say, it popped up again" (patient 8)

"Well, from my side, I'm gonna say yes, because after they did the radiation, I had, I can't tell you, I think I've... I had a scan, CT scan, [...], and anyway I got an answer back from Dr *** says that it appears that we had, I don't know the words he used but I got the impression that it was... we'd slowed down the spread of the cancer at that point in time" (patient 19)

"I was just, I mean I was just wiped out you know from... I had no energy [...] I spent a lot of time in bed, just lying there... I just didn't feel like doing anything. I had, you know, a little bit of nausea" (patient 16)

"During the chemo treatment, it slowed me down a little bit, I started getting fatigue on about the second day of the treatments" (patient 3)

"Radiation, I never had any kind of a side effect whatsoever, I was up and going right after it, not a problem" (patient 1)

"I'd go outside to do some work outside in the yard and after about 20 minutes or so, I'd say I gotta go sit down" (patient 19)

"I still walk and I still did everything I had to do but I didn't have the energy to do a lot more than that" (patient 8)

"It's slowed me down. I'm a very active person and we like to travel, probably abroad, and do things, but we haven't been able to travel, because of treatments" (patient 7)

"My sleep was crazy during chemo [...] I had chemo, and from that Wednesday after I had my first treatment on Tuesday, I would not sleep very well, and when I say very well, I'm talking about, you know, maybe an hour or so a night. Through Monday. And then Monday I crashed, I mean I would go down hard. Sleep an awful lot" (patient 3)

"Actually it hindered it more than it because it was so time consuming, and it did not, in my case, did not do anything except, you know, weaken you as an individual, health wise" (patient 1)

"I have high hopes, that it will be effective and mostly shrink the thing, and now I am hoping it will shrink, get rid of the ones that are in my body, distal metastasis" (patient 7)

"Well I'm hoping for a partial cure, something longer than a month or two at a time. A few more years on my life, it would help" (patient 8)

"You know, it may not work for everyone, but it certainly has had some spectacular results, and I'd love to see that myself, of course" (patient 17)

"You've covered a pretty wide gamut of information, you know, I think I'm excited about this new opportunity to fight cancer myself, versus having chemo" (patient 3) 


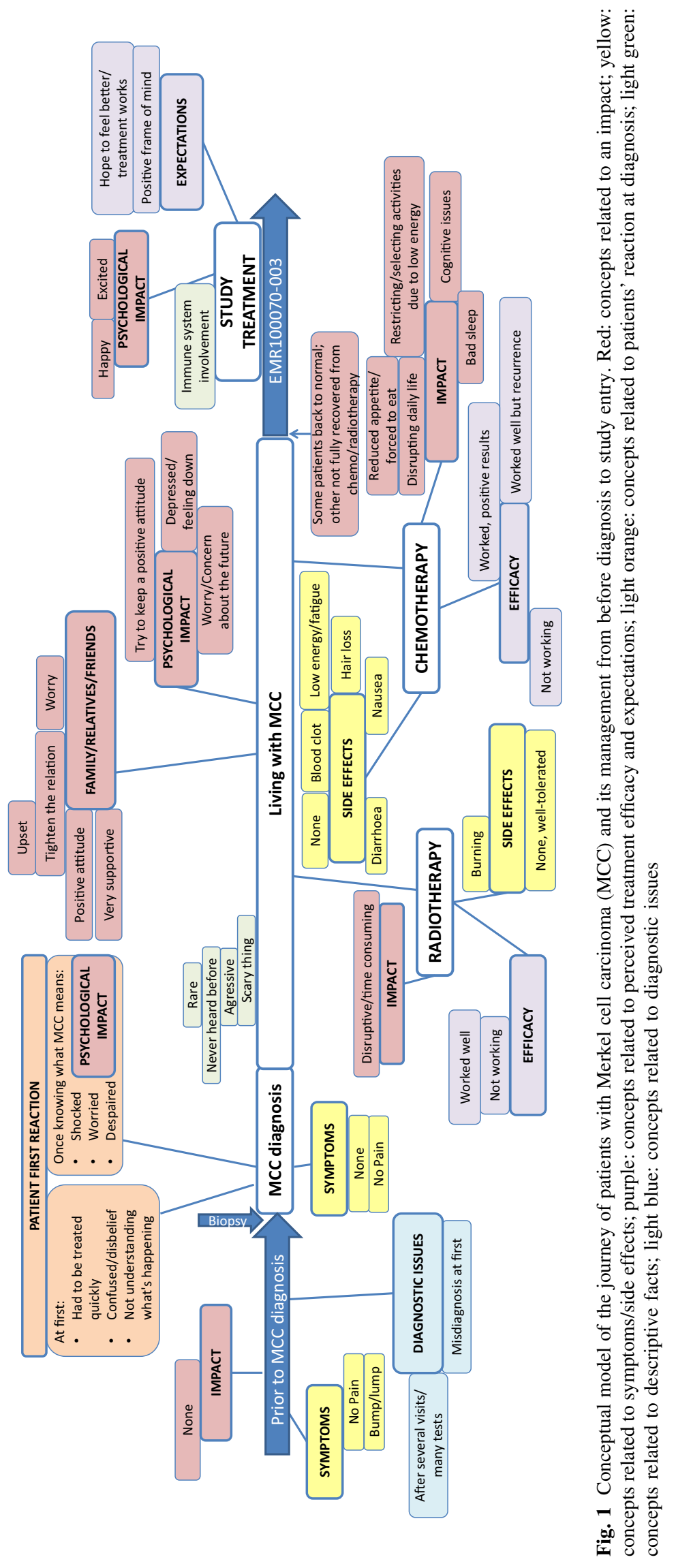




\section{Discussion}

Data from patient-reported outcome (PRO) measures or patient interviews are valuable not only for supporting findings from standard trial endpoints, but also for supporting the rationale for reimbursement decisions and defining the patient perspective on value for specific treatment regimens. Patient interviews and qualitative research help inform and provide an in-depth understanding of patients' experiences of both the condition and its treatment [19]. In addition, qualitative research can be integrated into clinical trials to support clinical findings [20]. In particular, qualitative research is of high value in the context of rare diseases when facing a small sample size. Because MCC is a rare disease, any study involving patients with MCC provides a good opportunity to gather additional information relating to disease impact and management. The present qualitative study was part of a phase 2 trial in patients with metastatic MCC who had failed previous chemotherapy [11]. The exploratory interviews conducted before the first administration of study treatment provided a comprehensive view of the impact of MCC and its management on patients' everyday lives prior to diagnosis, at diagnosis, and during treatment with chemotherapy and/or radiotherapy. The small proportion of patients interviewed, which could be seen as a limiting factor for the generalisability of the findings at first, should, however, be put into the perspective of the rarity of the disease, and therefore the interviews should be considered a valuable means of collecting information for a condition that is poorly documented to date.

Overall, MCC was perceived by patients as a rare and aggressive disease for which little information was available from either healthcare professionals or other sources. MCC was reported as being mostly painless and asymptomatic, both before and after diagnosis, and most reports of adverse impact or pain corresponded to the consequences of surgery to remove the tumour rather than the cancer itself.

Living with MCC was mainly described as being close to normal in terms of usual daily functioning; patients were still able to perform their activities of daily living and had full physical capabilities. However, a substantial psychological impact was reported. While patients did not fully understand the implications of MCC at first, after being informed about its seriousness, they felt shocked by the unexpected diagnosis and scared about the disease and its outcome.

The MCC tumour may be mistakenly considered to be benign and may often be misdiagnosed. This could be due, as patients noted, to the lack of knowledge and awareness of MCC by many physicians and health professionals. This could result in many referrals and delays in diagnosis, causing frustration and irritation in patients who perceive that their cancer could have been managed more quickly. These findings are somewhat consistent with those reported by patients with melanoma, who also face challenges in the early detection of skin changes and correct diagnosis [21].

In contrast to MCC itself, the side effects of treatment were often highly debilitating and substantially affected patients' everyday lives, both physically and psychologically. The lack of energy and fatigue associated with chemotherapy were particularly deleterious. The limited efficacy of chemotherapy and radiotherapy, reflected by frequent recurrence, added to the negative opinion of patients towards these treatments. In the absence of alternative treatment options, all patients expressed strong hopes and therapeutic optimism at study entry and prior to receiving avelumab.

This study has several limitations. First, the impact of MCC is closely intertwined with the impact of the treatment. Patients could not be certain about whether an impact was attributable to the cancer or its treatment. In particular, patients experiencing pain did not discern whether this was a disease-related or treatment-related symptom. In contrast, fatigue and lack of energy were associated with chemotherapy. The limited impact of MCC on patients' lives might have been underestimated. Similarly, the older age and retired status of most patients could have limited the perceived impact of the disease and treatment on their professional lives. Second, trial eligibility criteria mandated that all patients had undergone chemotherapy for their metastatic MCC and that their disease had progressed after the latest treatment. Thus, it is not surprising that only a subset of patients reported an initial benefit of chemotherapy, in terms of tumour shrinking or delayed progression, but most patients reported that chemotherapy did not have long-lasting effects. Limited durability of response to chemotherapy in MCC has been reported previously [22]. It is also acknowledged that our study population may be a specific group, not representative of the full MCC population; as not all patients participated in the interviews, selection bias cannot be excluded, e.g. patients who may have had differing experiences may have been less likely to volunteer.

Given the lack of published qualitative data about the lives of patients with MCC, how they perceive their cancer and its treatment, and how this cancer manifests, as well as the shortage of evidence-based treatments and sparse literature about MCC, this study should help improve awareness and understanding of MCC for healthcare professionals and others involved in patient care. The concepts identified in this qualitative study as being important to patients with MCC can help define the choice of PRO instruments that could be used in MCC trials to document 
treatment benefit from the patients' perspective. The dissemination of such PRO data to individuals involved in patient care and reimbursement decisions (e.g. healthcare professionals, payers, and advocacy groups) is vital to raise awareness and highlight the unmet needs of patients with MCC [23]. This is particularly important given that regulatory bodies are increasing their focus on patient perspectives [23]. The European Organisation for Research and Treatment of Cancer (EORTC) questionnaires and Functional Assessment of Cancer Therapy (FACT) questionnaires [24] are frequently used in oncology trials; however, no MCC-specific modules have been developed and validated to date. The present study could help with selecting relevant items from existing questionnaires to derive an MCC-relevant score, or could help to develop an MCC-specific instrument.

\section{Conclusions}

This qualitative research is the first of its kind to report data on MCC and its management from the patients' perspective. Overall, in our study sample, MCC disease was not perceived by the interviewed patients to result in severe functional limitations, nor to impact everyday activities, but was associated with substantial negative psychological impact. In contrast, chemotherapy and radiotherapy for MCC were described as highly debilitating and disrupting patients' lives. Further longitudinal analysis of follow-up interviews should help to document patient experiences with a novel treatment and could potentially document treatment benefit.

Acknowledgements The authors thank all participating patients and their families and the investigators, interviewers, and trial staff.

Data availability statement The datasets generated during and/or analysed during the current study are available from the corresponding author $(\mathrm{HK})$ on reasonable request.

Author contributions CDB, IG, JL, and MB contributed to the study design; IG and JL performed the analyses of interviews. All authors contributed to the interpretation of the data and critically reviewed the manuscript during its preparation and approved the final version.

\section{Compliance with Ethical Standards}

Funding This study was funded by and is part of an alliance between Merck KGaA and Pfizer, Inc, NY, USA.

Conflict of interest MB is an employee of Merck KGaA, Darmstadt, Germany. CDB and IG, employees of Mapi at the time the study was performed, and JL, an employee of Mapi, were paid consultants to Merck KGaA, Darmstadt, Germany. HK has served on advisory boards for Amgen, Celldex, Compass Therapeutics, EMD Serono, Merck, Prometheus, Sanofi and Turnstone Biologics. He has also served on a speakers' bureau for Merck, but all honoraria were given to Rutgers University. LM is an employee of EMD Serono, Boston, USA. The authors did not receive payment for authorship.

Informed consent All participants provided written informed consent prior to any study-related procedures, including patient interviews. The study was reviewed and approved by all local applicable institutional review boards and ethics committees.

Open Access This article is distributed under the terms of the Creative Commons Attribution-NonCommercial 4.0 International License (http://creativecommons.org/licenses/by-nc/4.0/), which permits any noncommercial use, distribution, and reproduction in any medium, provided you give appropriate credit to the original author(s) and the source, provide a link to the Creative Commons license, and indicate if changes were made.

\section{References}

1. Becker JC. Merkel cell carcinoma. Ann Oncol. 2010;21(Suppl 7):vii81-5. https://doi.org/10.1093/annonc/mdq366.

2. Oram CW, Bartus CL, Purcell SM. Merkel cell carcinoma: a review. Cutis. 2016;97(4):290-5.

3. Spurgeon ME, Lambert PF. Merkel cell polyomavirus: a newly discovered human virus with oncogenic potential. Virology. 2013;435(1):118-30. https://doi.org/10.1016/j.virol.2012.09.029.

4. Wong SQ, Waldeck K, Vergara IA, Schroder J, Madore J, Wilmott JS, et al. UV-associated mutations underlie the etiology of MCV-negative Merkel cell carcinomas. Cancer Res. 2015;75(24):5228-34. https://doi.org/10.1158/0008-5472.CAN15-1877.

5. Goon PK, Greenberg DC, Igali L, Levell NJ. Merkel cell carcinoma: rising incidence in the east of England. J Eur Acad Dermatol Venereol. 2016. https://doi.org/10.1111/jdv.13828.

6. Zaar O, Gillstedt M, Lindelof B, Wennberg-Larko AM, Paoli J. Merkel cell carcinoma incidence is increasing in Sweden. J Eur Acad Dermatol Venereol. 2016. https://doi.org/10.1111/jdv. 13698.

7. Fitzgerald TL, Dennis S, Kachare SD, Vohra NA, Wong JH, Zervos EE. Dramatic increase in the incidence and mortality from Merkel cell carcinoma in the United States. Am Surgeon. 2015;81(8):802-6.

8. National Comprehensive Cancer Network website. NCCN clinical practice guidelines in oncology, Merkel cell carcinoma, version 1. 2017. http://www.nccn.org. Accessed 12 Oct 2017.

9. Soult MC, Feliberti EC, Silverberg ML, Perry RR. Merkel cell carcinoma: high recurrence rate despite aggressive treatment. J Surg Res. 2012;177(1):75-80. https://doi.org/10.1016/j.jss. 2012.03.067.

10. Lebbe C, Becker JC, Grob JJ, Malvehy J, Del Marmol V, Pehamberger $\mathrm{H}$, et al. Diagnosis and treatment of Merkel cell carcinoma. European consensus-based interdisciplinary guideline. Eur J Cancer. 2015;51(16):2396-403. https://doi.org/10. 1016/j.ejca.2015.06.131.

11. Kaufman HL, Russell J, Hamid O, Bhatia S, Terheyden P, D'Angelo SP, et al. Avelumab in patients with chemotherapyrefractory metastatic Merkel cell carcinoma: a multicentre, single-group, open-label, phase 2 trial. Lancet Oncol. 2016. https:// doi.org/10.1016/S1470-2045(16)30364-3.

12. Nghiem PT, Bhatia S, Lipson EJ, Kudchadkar RR, Miller NJ, Annamalai L, et al. PD-1 blockade with pembrolizumab in advanced Merkel-cell carcinoma. N Engl J Med. 2016;374(26):2542-52. https://doi.org/10.1056/NEJMoa1603 702 . 
13. Grant A. Fear, confusion and participation: Incapacity Benefit claimants and (compulsory) Work Focused Interviews. Res Policy Plan. 2011;28(3):161-71.

14. Sweet L. Telephone interviewing: is it compatible with interpretive phenomenological research? Contemp Nurse. 2002;12(1):58-63.

15. Friese S. ATLAS.ti 7 user guide and reference. Berlin, Germany. 2014 http://atlasti.com/wp-content/uploads/2014/05/atlasti_v7_ manual_201312.pdf?q=/uploads/media/atlasti_v7_manual_ 201312.pdf. Accessed 5 Jan 2016.

16. Lewins A, Silver C. Using software in qualitative research: a step-by-step guide. London: SAGE Publications; 2007.

17. Boyatzis R. Transforming qualitative information: thematic analysis and code development. Thousand Oaks: Sage; 1998.

18. US Department of Health and Human Services, Food and Drug Administration, Center for Drug Evaluation and Research, Center for Biologics Evaluation and Research, Center for Devices and Radiological Health. Guidance for industry. Patient-reported outcome measures: use in medical product development to support labeling claims. 2009. https://www.fda.gov/downloads/ drugs/guidances/ucm193282.pdf. Accessed 1 Mar 2018.

19. Britten N. Qualitative research on health communication: what can it contribute? Patient Educ Couns. 2011;82(3):384-8. https:// doi.org/10.1016/j.pec.2010.12.021.
20. O'Cathain A, Thomas KJ, Drabble SJ, Rudolph A, Hewison J. What can qualitative research do for randomised controlled trials? A systematic mapping review. BMJ Open. 2013. https://doi. org/10.1136/bmjopen-2013-002889.

21. Walter FM, Birt L, Cavers D, Scott S, Emery J, Burrows N, et al. 'This isn't what mine looked like': a qualitative study of symptom appraisal and help seeking in people recently diagnosed with melanoma. BMJ Open. 2014;4(7):e005566. https://doi.org/10. 1136/bmjopen-2014-005566.

22. Iyer J, Blom A, Doumani R, Lewis C, Anderson A, Ma C et al., editors. Response rate and durability of chemotherapy for metastatic Merkel cell carcinoma among 62 patients. In: American Society of Clinical Oncology (ASCO) 50th annual meeting; 2014 30/05/2014 to 03/06/2014; Chicago, IL.

23. Hao Y, Krohe M, Yaworsky A, Shields AL, Mazar I, Foley C, et al. Clinical trial patient-reported outcomes data: going beyond the label in oncology. Clin Ther. 2016;38(4):811-20. https://doi. org/10.1016/j.clinthera.2016.03.010.

24. Luckett T, King MT, Butow PN, Oguchi M, Rankin N, Price MA, et al. Choosing between the EORTC QLQ-C30 and FACT-G for measuring health-related quality of life in cancer clinical research: issues, evidence and recommendations. Ann Oncol. 2011;22(10):2179-90. https://doi.org/10.1093/annonc/mdq721. 